\title{
Camera Self-Calibration with Varying Intrinsic Parameters by an Unknown Three-Dimensional Scene
}

\author{
B. SATOURI \\ LIIAN, Department of Mathematics and informatics \\ Faculty of Sciences Dhar-Mahraz P.O.Box 1796 Atlas- Fes, \\ Morocco
}

\section{A. EL ABDERRAHMANI}

LIIAN, Department of Mathematics and informatics Larache Poly disciplinary School, LARACHE, Morocco

\author{
H. TAIRI \\ LIIAN, Department of Mathematics and informaticsFaculty of \\ Sciences Dhar-Mahraz P.O.Box 1796 Atlas- Fes, Morocco \\ K. SATORI \\ LIIAN, Department of Mathematics and informatics \\ Faculty of Sciences Dhar-Mahraz P.O.Box 1796 Atlas- Fes, \\ Morocco
}

\begin{abstract}
In the present paper, we will propose a new and robust method of camera self-calibration having varying intrinsic parameters from a sequence of images of an unknown $3 D$ object. The projection of two points of the 3D scene in the image planes is used to determine the projection matrices. The present method is based on the formulation of a non linear cost function from the determination of a relationship between two points of the scene with their opposite relative to the axis of abscise and their projections in the image planes. The resolution of this function with genetic algorithm enables us to estimate the intrinsic parameters of different cameras. The important of our approach reside in the use of a single pair of images which provides fewer equations, simplifies the mathematical complexities and minimizes the execution time of the application, the use of the data of the first image only without the use of the data of the second image, the use of any camera which makes the intrinsic parameters variable not constant and the use of a 3D scene reduces the planarity constraints. The experimental results on synthetic and real data prove the performance and robustness of our approach.
\end{abstract}

Keywords-Self-calibration; varying intrinsic parameters; non linear optimization; Interests points; Matching; Fundamental matrix

\section{INTRODUCTION}

Computer vision is the science of vision machines. It is a scientist discipline who is interested in building artificial systems that obtain information from images. The input data can take many forms: photographs, video footage, multiple camera images or multidimensional data medical scanner. Subdomains of computer vision are for example the Reconstruction of scenes, detection of events, object recognition, learning and image restoration.

The Reconstruction of 3D scenes is a research path which became very important and active with the advent of visualization by computer. As a matter of fact this technique will be found in various fields almost all of them situated on the crossroads of IT(data processing), mathematics and some of robotics related disciplines. The major objectif is always to extract information on the three-dimensional scene from a set of images gathered by numerical cameras with or without a priori knowledge of the scene. Therefore it will become clear and necessary to begin by modeling the camera. The parameters of the cameras can be estimated by two major methods: calibration [1, 2, 3, 4] and self-calibration. In this paper, we are interested in the self-calibration methods that can calibrate the cameras without any prior knowledge about the scene. The standard process of most of these methods is to search for equations according to intrinsic parameters and the invariants in the images, whose aim generally is to solve a nonlinear equation system. The algorithm used to solve this system requires two steps, initialization and optimization of a cost function. Self-calibration of the cameras is the main step to obtain three-dimensional coordinates of points from matches between pairs of images. Several methods of camera self-calibration with constant intrinsic parameters [5-14] and those with varying intrinsic parameters [15-25] are treated in this area.

Our approach is a new and robust method for camera selfcalibration having the varying intrinsic parameters by the use of an unknown three-dimensional scene. After the detection of interests points in the images by the Harris method [26] and the matching of these points in each pair of images by the correlation measure ZNCC [27], the fundamental matrix can be estimated from eight matches by the RANSAC algorithm [28]. This matrix is used with the projection of four points of the 3D scene in images taken by different views in order to formulate linear equations. Solving these equations allows the estimation of the projection matrices. The determination of a relationship between the four points of the 3D scene and their projections in the planes of the images $\mathrm{g}$ and $\mathrm{d}$ and the relationships between the images of the absolute conic allow the formulation of a nonlinear cost function. The minimization of this function by the genetic algorithms [29]allows the estimation of the intrinsic parameters of the cameras used.

Our method presents a novelty: two images only are sufficient to estimate the cameras' intrinsic parameters, the use of the data of the first image only, the use of any camera (with varying intrinsic parameters) and the use of an unknown 3D scene. These advantages allow us, on the one hand, to solve some problems related to the self-calibration system and, on the other hand, to work freely in the domain of selfcalibration with fewer constraints. 
Our work is organized as follows: In the third part, we present the camera model and matching containing three subparts: The first subpart comprises the camera model, the second is the Interest points 'detection. It is a preliminary step in many computer vision processes; many methods have been advanced to extract points of interest. In this paper, we used Harris interest point detector. The third is the Matching: Finding in two images of the same scene, taken at different positions, pairs of pixels which are the projections of the same point of the scene. In this phase, the detected interest points are matched by ZNCC (Zero mean Normalized Cross Correlation) correlation measure. The most important section is related to the estimation of the projection matrices and selfcalibration equations in section four and five. The experiment results are discussed in the Seven part, and finally, in section ten we will proceed to make a general conclusion.

\section{SURVEY OF THE PREVIOUS WORKS}

In order to make the self-calibration with the intrinsic constant parameters some of the concepts are based on simplified models which are designed to make the equations less complex which often allows them to converge into good results [30,31] Others are based on particular movements of the camera [32,33]. In there is also the category of the concepts which exploit general movements of constant intrinsic parameters [7] that takes into account cyclical points within a key view, in a flat scene and homographies. Other authors have shown various studies based on the Kruppa equations $[34,35]$ which on the one hand simplify the selfcalibration process by a direct estimation without making a projective reconstruction and on the other one by eliminating the infinite plane (the projection matrices have disappeared and only the fundamental matrices and the epipoles are present). In the same context other practical methods are proposed: [36] a method which presents an analytical reduction of the Kruppa equations. [37] This article presents a framework for random sampling nonlinear optimization for the self-calibration with modeling intrinsic parameters space of the camera, the focal length is modeled by using a Gaussian distribution originated by the Kruppa equations while the optical center is close to the center of the picture, this model allows the cost of the calculations.

[38] This article deals with the problem of self- calibrating a moving camera with constant parameters. This method proposes a new set of quartic trivariate polynomial equations within the unknown coordinates of the indefinite plane derived from the hypothesis of no-skew, these new equations allow to better respect the constant of the principal point in all the images when recuperating the infinite plan. [39] In the present article a new method that combines the parallelism plane and the self-calibrating constraints of Mendonça/Cipolla. In this technique each pair of images is used independently and therefore presents a pair of different parallel planes not necessarily visible in the other images.

In order to solve the problem of the self-calibration of the cameras our interest nowadays goes to the category of concepts that exploit the varying intrinsic parameters. Amongst them we recognize various approaches. One of them [40] consists of the determination of the dual absolute quadric of which the image is the dual absolute conic. The idea is then to transfer these constraints to the dual absolute quadric; once this matrices is known it suffices to determine the transformation that will replace the dual absolute in its canonical position. [41] A recent analogue method consists in the use of the dual absolute line (ALQ) instead of the absolute conic. [42, 43] They have solved the problem of the selfcalibration through the use of Kruppa equations with the study of the varying intrinsic parameters case.

[44] A new method of self-calibration and stratified metric reconstruction for zooming/refocusing cameras is proposed sticking to the circular movement and its constraints: the ambiguity is then solved with the hypothesis of the square pixel of the camera and this flexibility allows the focal length and the principal point to vary. [45] This article details a method of self-calibration of the varying internal parameters of the camera that is based on a dual absolute quadric transformation of the image. This method may lead to a considerable improvement of the stability and robustness of the results. [46] This article describes a new method for the self-calibration of a sequence of images with the varying intrinsic parameters of the camera. This article is based on the Kruppa equations. This technique is based on the Kruppa equations with two upper triangular matrixes with which a relational matrix should be in place. Utilizing this way the epipolar geometry relationship of absolute conic to obtain the intrinsic parameters of the camera. [47] This article proposes a new method of initialization using a minimum of two images. The basic idea is that the minimum deviation of the intrinsic parameter will result in a more stable result. [48] This article presents an algorithm of sequential filtering to reach a simulated estimation of the 3D scenes. The auto calibration in this article uses the standard projective parameters of the focal distance and the principal point with two coefficients of the radial distortion. [49] A solid linear method for the selfcalibration of a moving camera starting from a sequence of images is presented. The proposed approach uses known linear equations which are weighted by variable factors. The experiments show that this modification reduces the problems with the critical motion sequences.

\section{CAMERA MODEL AND MATCHING}

\section{A. Pinhole Camera Model}

The pinhole model (Figure 1) projects the scene in the image planes, for the camera g, it is defined by $K_{g}\left(R_{g} t_{g}\right)$ with a matrix $\left(R_{g} t_{g}\right)$ containing extrinsic parameters $R_{g}$ the rotation matrix, and $\mathrm{t}_{\mathrm{g}}$ the translation vector of camera in space, $\mathrm{K}$ gis a matrix containing the intrinsic parameters and is expressed as follows:

$$
K_{g}=\left(\begin{array}{ccc}
f_{g} & \tau=0 & u_{0 g} \\
0 & \varepsilon_{g} f_{g} & v_{0 g} \\
0 & 0 & 1
\end{array}\right)
$$

\footnotetext{
$f_{g}$ is the focal length

$\varepsilon_{g}$ is the scale factor
} 
$\tau$ is the skew factor

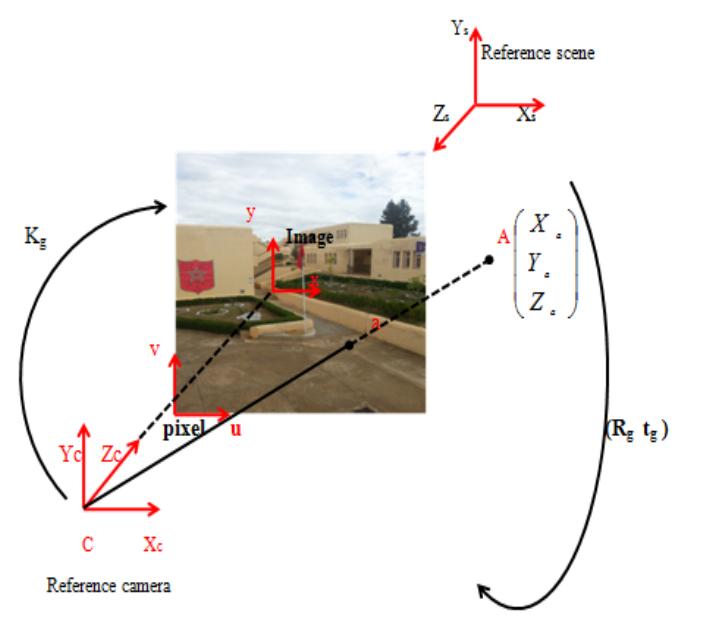

Fig. 1. Pinhole camera model

\section{B. Interests points}

Interest points are characteristic points of the image that are particularly holders of information. The detection of these points in stereoscopic images is an essential step in the field of Computer Vision and especially in the Three-dimensional Reconstruction. It is to match the projections of the same entity in the scene. The pairing of the corners points is based on the calculation and evaluation of degree of similarity of two pixel areas. For a pixel and its vicinity in the left image, we look at the other image pixel and its neighborhood that suits them the most. This step requires robust algorithms vis-avis different geometrical transformations and changes in illuminance disturbances.

We begin by extracting the corners points with the Harris detector that exists in the literature:

\section{1) Detection Of Interest Points}

The detectors of interests points found in the literature are: Moravec detector [27], Harris detector, Susan detector [28],... Harris [23],[26] developed the method Moravec for calculating local maxima in the images used by a matrix $\mathrm{N}$ related to the autocorrelation function which takes into account the first derivatives of the values of the signal I on a window in image space. $\mathrm{N}$ matrix is calculated by the following formula:

$$
\mathrm{N}=\left(\begin{array}{cc}
\left(\frac{\partial I}{\partial u}\right)^{2} & \left(\frac{\partial I}{\partial u}\right)\left(\frac{\partial I}{\partial v}\right) \\
\left(\frac{\partial I}{\partial u}\right)\left(\frac{\partial I}{\partial v}\right) & \left(\frac{\partial I}{\partial v}\right)^{2}
\end{array}\right)
$$

In order not to extract the values of this matrix, Harris uses a variable $r$ whish is greater than zero in the case of a corner(interest point), its value is given by:

$$
\mathrm{r}=\operatorname{det}(N)-\gamma[\operatorname{trace}(N)]^{2}
$$

$\left(\begin{array}{ll}u_{0 g} & v_{0 g}\end{array}\right)$ represent the coordinates of the principal point in the images.

$$
\gamma=0.04 \text { (Value fixed by Harris) }
$$

The detected primitive type is given by the values of $r$, three cases:

$\mathrm{r}<0$ : in the vicinity of an edge

$\mathrm{r}=0$ : in a homogeneous region

r > threshold: near a point of corners

\section{2) Correlation Measure}

Corresponding points between two images of the sequence are the points of Harris previously detected in each image and matched by the correlation measure ZNCC(Zero mean Normalized Cross Correlation) [24], [25] which is invariant to local linear change luminance .m and m' are two interest points detected in the left and right image respectively. Measurement correlation $\mathrm{ZNCC}(\mathrm{m}, \mathrm{m}$ ') is given by the formula follows:

$$
\operatorname{ZNCC}\left(\mathrm{m}, \mathrm{m}^{\prime}\right)=\frac{\sum_{i}\left((I(m+i)-\bar{I}(m))\left(I^{\prime}\left(m^{\prime}+i\right)-\overline{I^{\prime}}\left(m^{\prime}\right)\right)\right)}{\sum_{i}(I(m+i)-\bar{I}(m))^{2} \sum_{i}\left(I^{\prime}\left(m^{\prime}+i\right)-\overline{I^{\prime}}\left(m^{\prime}\right)\right)^{2}}
$$

With I (m) and I'(m') the average luminance of pixels in a window of size $11 \times 11$ respectively in $\mathrm{m}$ and $\mathrm{m}$ ' and $\mathrm{i}$ varies from 1 to $n$.

\section{Estimation OF THE PROJECTION MATRICES}

Considering two points $A_{1}, A_{2}$ and their opposite $A_{3}, A_{4}$ relative to the axis of abscise on the $3 \mathrm{D}$ scene. Let $\pi$ a plane that contains these four points, we consider $\mathrm{R}$ an euclidean reference ( $\mathrm{O} X \mathrm{Y} \mathrm{Z}$ ) such as $\mathrm{O}$ is the midpoint of the chord $\left[\mathrm{A}_{1} \mathrm{~A}_{2}\right]$, and $\mathrm{Z}$ is perpendicular on the plan of the scene $(Z \perp \pi)$. The homogeneous coordinates of the four points $A_{1}, A_{2}, A_{3}$ and $A_{4}$ (Figure 2) in the reference R (O X Y)are given as follows:

with 


$$
\begin{aligned}
& A_{1}=(d \cos \varphi, d \sin \varphi, 1)^{T} \\
& A_{2}=(-d \cos \varphi,-d \sin \varphi, 1)^{T} \\
& A_{3}=(d \cos \varphi,-d \sin \varphi, 1)^{T} \\
& A_{4}=(-d \cos \varphi, d \sin \varphi, 1)^{T}
\end{aligned}
$$

Where $\mathrm{d}=\mathrm{A}_{1} \mathrm{~A}_{2} / 2$ and $\varphi$ is the angle between the chord $\left[\mathrm{A}_{1} \mathrm{~A}_{2}\right]$ and the $X$-axis. Considering two homographies $\mathrm{H}_{\mathrm{g}}$ and $\mathrm{H}_{\mathrm{d}}$ that can project the plane $\pi$ in images $\mathrm{g}$ and $\mathrm{d}$, therefore, the projection of the four points $A_{1}, A_{2}, A_{3}$ and $A_{4}$ can be given by the following expressions:

$$
a_{g k}=H_{g} A_{k}
$$

Where $\mathrm{k}=1,2,3,4$ and $\mathrm{a}_{\mathrm{gk}}$ represent the points in the images $g$ that are the projections of the four vertices $A_{1}, A_{2}$, $A_{3}$ and $A_{4}$ of the $3 D$ scene, and $H_{g}$ represent the homography matrices that are expressed as follows:

$$
H_{g} \sim \mathrm{K}_{\mathrm{g}} R_{g}\left(\begin{array}{ccc}
1 & 0 & \\
0 & 1 & R_{g}^{T} t_{g} \\
0 & 0 &
\end{array}\right)
$$

Expressions (5) can be written as follows:

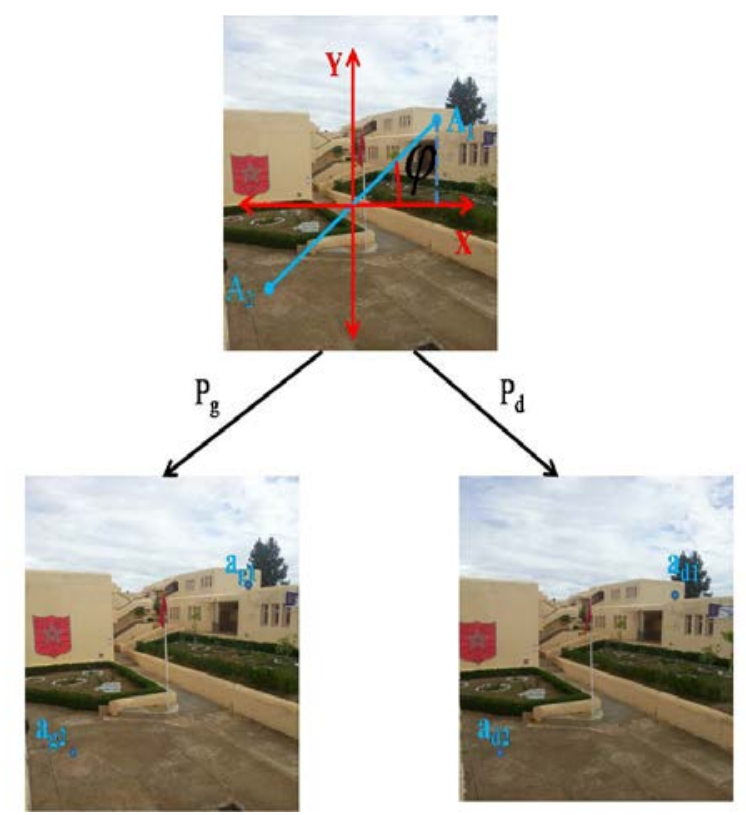

Fig. 2. The projection of the two points $A_{1}, A_{2}$ in the planes of images $g$ and $d$

Expression (9) gives:

$$
\begin{aligned}
a_{g 1} & \sim \mathrm{P}_{\mathrm{g}} A_{1}^{\prime} \\
a_{g 2} & \sim \mathrm{P}_{\mathrm{g}} A_{2}^{\prime} \\
a_{g 3} & \sim \mathrm{P}_{\mathrm{g}} A_{3}^{\prime} \\
a_{g 4} & \sim \mathrm{P}_{\mathrm{g}} A_{4}^{\prime}
\end{aligned}
$$

$$
a_{g k} \sim \mathrm{H}_{\mathrm{g}} M A_{k}^{\prime}
$$

$$
\begin{aligned}
& \text { Where } M=\left(\begin{array}{ccc}
d \cos \varphi & 0 & 0 \\
0 & d \sin \varphi & 0 \\
0 & 0 & 1
\end{array}\right) \text { and } \\
& \qquad A_{k}^{\prime}=\left(\begin{array}{c}
m_{1} \\
m_{2} \\
1
\end{array}\right) \quad\left\{\begin{array}{llll}
k=1 & & m_{1}=1 & m_{2}=1 \\
k=2 & \Leftrightarrow & m_{1}=-1 & m_{2}=-1 \\
k=3 & & m_{1}=1 & m_{2}=-1 \\
k=4 & & m_{1}=-1 & m_{2}=1
\end{array}\right\}
\end{aligned}
$$

We can represent the projection matrices by:

$$
P_{g} \sim \mathrm{H}_{\mathrm{g}} M
$$

Where $\mathrm{P}_{\mathrm{g}}$ represent the projection matrices of the four points $A^{\prime}{ }_{1}, A^{\prime}{ }_{2}, A^{\prime}{ }_{3}, A^{\prime}{ }_{4}$ in images (Figure 2).

Formula (8) give:

$$
a_{g k} \sim \mathrm{P}_{g} A_{k}^{\prime}
$$

The latter four relations give eight equations with eight unknowns, which are the $\mathrm{P}_{\mathrm{g}}$ elements.

So, the $\mathrm{P}_{\mathrm{g}}$ parameters can be estimated from these eight equations with eight unknowns.

\section{SELF-CALIBRATION EQUATIONS}

A nonlinear cost function will be defined in the main part of this work from the determination of the relationships 
between the images of the absolute conic $\left(\omega_{g}\right)$ and from the relationships between two points $\left(\mathrm{A}_{1}, \mathrm{~A}_{2}\right)$ and his oppositely relative to the $\mathrm{X}$ axis $\left(\mathrm{A}_{3}, \mathrm{~A}_{4}\right)$ of the3D scene and their projections $\left(A_{g 1}, A_{g 2}, A_{g 3}, A_{g 4}\right)$ and in the planes of the images g, respectively. The different relationships are based on some techniques of projective geometry. The defined cost function will be minimized by the genetic algorithms to estimate the $\omega_{g}$ elements and, finally, by the intrinsic parameters of the cameras used.

Expression (9) gives

Where

$$
\alpha_{g k} a_{g k}=P_{g} A_{k}^{\prime}
$$

$P_{g}=\left(\begin{array}{lll}P_{g 11} & P_{g 12} & P_{g 13} \\ P_{g 21} & P_{g 22} & P_{g 23} \\ P_{g 31} & P_{g 32} & P_{g 33}\end{array}\right), \quad a_{g k}=\left(\begin{array}{c}x_{g k} \\ y_{g k} \\ 1\end{array}\right) \quad$ and

$\alpha_{g k}=m_{1} P_{g 31}+m_{2} P_{g 32}+P_{g 33}$

$\alpha_{g k}$ is a nonzero scale factor that is used to realized the transition between equality with a scale factor $\sim$ to precise equality $=$. The value of $\alpha_{g k}$ is determined from expression (11).

Therefore, formula (11) leads to:

$$
\alpha_{g k} a_{g k}^{\prime}=P_{g} A_{k}^{\prime \prime}
$$

Where

$$
a_{g k}^{\prime}=\left(\begin{array}{ccc}
x_{g k} & \frac{P_{g 12}}{\alpha_{g k}} & \frac{P_{g 13}}{\alpha_{g k}} \\
y_{g k} & \frac{P_{g 22}}{\alpha_{g k}} & \frac{P_{g 23}}{\alpha_{g k}} \\
1 & \frac{P_{g 32}}{\alpha_{g k}} & \frac{P_{g 33}}{\alpha_{g k}}
\end{array}\right) \text { and } A_{k}^{\prime \prime}=\left(\begin{array}{ccc}
m_{1} & 0 & 0 \\
m_{2} & 1 & 0 \\
1 & 0 & 1
\end{array}\right)
$$

Expression (12) gives:

$$
P_{g} \sim a_{g k}^{\prime} A_{k}^{\prime \prime-1}
$$

Expressions (6) and (8) give:

$$
P_{g} \sim K_{g} R_{g}\left(\begin{array}{ccc}
m_{1} & 0 & \\
m_{2} & 1 & R_{g}^{T} t_{g} \\
1 & 0 &
\end{array}\right) M
$$

The previous formula leads to:

$$
K_{g}^{-1} P_{g} \sim R_{g}\left(\begin{array}{ccc}
m_{1} & 0 & \\
m_{2} & 1 & R_{g}^{T} t_{g} \\
1 & 0 &
\end{array}\right) M
$$

According to the formula (15) we have:

$$
P_{g}^{T} \omega_{g} P_{g} \sim\left(\begin{array}{cc}
M^{\prime T} M^{\prime} & M^{T} R_{g}^{T} t_{g} \\
t_{g}^{T} R_{g} M^{\prime} & t_{g}^{T} t_{g}
\end{array}\right)
$$

Where $\omega_{g}=\left(K_{g} K_{g}^{T}\right)^{-1}$ is the image of the absolute conic, and $M^{\prime}=\left(\begin{array}{cl}d \cos \varphi & 0 \\ 0 & d \sin \varphi \\ 0 & 0\end{array}\right)$

Expressions (13) and (16) give:

$$
\left(a_{g k}^{\prime} A_{k}^{\prime \prime-1}\right)^{T} \omega_{g}\left(a_{g k}^{\prime} A_{k}^{\prime \prime-1}\right) \sim\left(\begin{array}{cc}
M^{\prime T} M^{\prime} & M^{T} R_{g}^{T} t_{g} \\
t_{g}^{T} R_{g} M^{\prime} & t_{g}^{T} t_{g}
\end{array}\right)
$$

The previous expression gives:

$$
\begin{aligned}
& \left(a_{g 1}^{\prime} A_{1}^{\prime \prime-1}\right)^{T} \omega_{g}\left(a_{g 1}^{\prime} A_{1}^{\prime \prime-1}\right) \sim\left(\begin{array}{cc}
M^{\prime T} M^{\prime} & M^{\prime T} R_{g}^{T} t_{g} \\
t_{g}^{T} R_{g} M^{\prime} & t_{g}^{T} t_{g}
\end{array}\right) \\
& \left(a_{g 2}^{\prime} A_{2}^{\prime \prime-1}\right)^{T} \omega_{g}\left(a_{g 2}^{\prime} A_{2}^{\prime \prime-1}\right) \sim\left(\begin{array}{cc}
M^{\prime T} M^{\prime} & M^{\prime T} R_{g}^{T} t_{g} \\
t_{g}^{T} R_{g} M^{\prime} & t_{g}^{T} t_{g}
\end{array}\right) \\
& \left(a_{g 3}^{\prime} A_{3}^{\prime \prime-1}\right)^{T} \omega_{g}\left(a_{g 3}^{\prime} A_{3}^{\prime \prime-1}\right) \sim\left(\begin{array}{cc}
M^{\prime T} M^{\prime} & M^{\prime T} R_{g}^{T} t_{g} \\
t_{g}^{T} R_{g} M^{\prime} & t_{g}^{T} t_{g}
\end{array}\right) \\
& \left(a_{g 4}^{\prime} A_{4}^{\prime \prime-1}\right)^{T} \omega_{g}\left(a_{g 4}^{\prime} A_{4}^{\prime \prime-1}\right) \sim\left(\begin{array}{cc}
M^{\prime T} M^{\prime} & M^{\prime T} R_{g}^{T} t_{g} \\
t_{g}^{T} R_{g} M^{\prime} & t_{g}^{T} t_{g}
\end{array}\right)
\end{aligned}
$$

The expressions (18), (19), (20) and (21) give:

$$
\begin{aligned}
& \qquad\left(a_{g 1}^{\prime} A_{1}^{\prime \prime-1}\right)^{T} \omega_{g}\left(a_{g 1}^{\prime} A_{1}^{\prime \prime-1}\right) \sim\left(a_{g 2}^{\prime} A_{2}^{\prime \prime-1}\right)^{T} \omega_{g}\left(a_{g 2}^{\prime} A_{2}^{\prime \prime-1}\right) \sim \\
& \left(a_{g 3}^{\prime} A_{3}^{\prime \prime-1}\right)^{T} \omega_{g}\left(a_{g 3}^{\prime} A_{3}^{\prime \prime-1}\right) \sim\left(a_{g 4}^{\prime} A_{4}^{\prime \prime-1}\right)^{T} \omega_{g}\left(a_{g 4}^{\prime} A_{4}^{\prime \prime-1}\right) \quad \text { (22) } \\
& \text { Let }
\end{aligned}
$$

$B=\left(\begin{array}{lll}b_{11 g} & b_{12 g} & b_{13 g} \\ b_{21 g} & b_{22 g} & b_{23 g} \\ b_{31 g} & b_{32 g} & b_{33 g}\end{array}\right)$ denotes the matrix corresponding to

$\left(a_{g 1}^{\prime} A_{1}^{\prime \prime}-1\right)^{T} \omega_{g}\left(a_{g 1}^{\prime} A_{1}^{\prime \prime}-1\right)$

$C=\left(\begin{array}{lll}c_{11 g} & c_{12 g} & c_{13 g} \\ c_{21 g} & c_{22 g} & c_{23 g} \\ c_{31 g} & c_{32 g} & c_{33 g}\end{array}\right)$ denotes the matrix corresponding to

$\left(a_{g 2}^{\prime} A_{2}^{\prime \prime-1}\right)^{T} \omega_{g}\left(a_{g 2}^{\prime} A_{2}^{\prime \prime-1}\right)$

$D=\left(\begin{array}{lll}d_{11 g} & d_{12 g} & d_{13 g} \\ d_{21 g} & d_{22 g} & d_{23 g} \\ d_{31 g} & d_{32 g} & d_{33 g}\end{array}\right)$ denotes the matrix corresponding to

$\left(a_{g 3}^{\prime} A_{3}^{\prime \prime}-1\right)^{T} \omega_{g}\left(a_{g 3}^{\prime} A_{3}^{\prime \prime-1}\right)$ 
$E=\left(\begin{array}{lll}e_{11 g} & e_{12 g} & e_{13 g} \\ e_{21 g} & e_{22 g} & e_{23 g} \\ e_{31 g} & e_{32 g} & e_{33 g}\end{array}\right)$ denotes the matrix corresponding to

$\left(a_{g 4}^{\prime} A_{4}^{\prime \prime-1}\right)^{T} \omega_{g}\left(a_{g 4}^{\prime} A_{4}^{\prime \prime-1}\right)$

and $M^{\prime T} M^{\prime}=\left(\begin{array}{cc}d^{2} \cos ^{2} \varphi & 0 \\ 0 & d^{2} \sin ^{2} \varphi\end{array}\right)$

Therefore, the formula (22) with the previous gives:

$$
\left\{\begin{array}{l}
b_{12 g}=0, \\
c_{12 g}=0, \\
d_{12 g}=0, \\
e_{12 g}=0, \\
\frac{b_{11 g}}{b_{13 g}}=\frac{c_{11 g}}{c_{13 g}}=\frac{d_{11 g}}{d_{13 g}}=\frac{e_{11 g}}{e_{13 g}} \\
\frac{b_{13 g}}{b_{22 g}}=\frac{c_{13 g}}{c_{22 g}}=\frac{d_{13 g}}{d_{22 g}}=\frac{e_{13 g}}{e_{22 g}} \\
\frac{b_{22 g}}{b_{23 g}}=\frac{c_{22 g}}{c_{23 g}}=\frac{d_{22 g}}{d_{23 g}}=\frac{e_{22 g}}{e_{23 g}} \\
\frac{b_{23 g}}{b_{33 g}}=\frac{c_{23 g}}{c_{33 g}}=\frac{d_{23 g}}{d_{33 g}}=\frac{e_{23 g}}{e_{33 g}}
\end{array}\right.
$$

The previous equations contain twenty eight equations with five unknowns that are the elements of $\omega_{g}$. This system is non-linear. So to solve it, we try to minimize the objective function with the genetic algorithms:

$$
\left\{\begin{array}{l}
b_{12 g}=0, \quad c_{12 g}=0, \quad d_{12 g}=0, \quad e_{12 g}=0, \\
b_{11 g} c_{13 g}-c_{11 g} b_{13 g}=0, \quad b_{11 g} d_{13 g}-d_{11 g} b_{13 g}=0, \quad b_{11 g} e_{13 g}-e_{11 g} b_{13 g}=0, \\
c_{11 g} d_{13 g}-d_{11 g} c_{13 g}=0, \quad c_{11 g} e_{13 g}-e_{11 g} c_{13 g}=0, \quad d_{11 g} e_{13 g}-e_{11 g} d_{13 g}=0, \\
b_{13 g} c_{22 g}-c_{13 g} b_{22 g}=0, \quad b_{13 g} d_{22 g}-d_{13 g} b_{22 g}=0, \quad b_{13 g} e_{22 g}-e_{13 g} b_{22 g}=0, \\
c_{13 g} d_{22 g}-d_{13 g} c_{22 g}=0, \quad c_{13 g} e_{22 g}-e_{13 g} c_{22 g}=0, \quad d_{13 g} e_{22 g}-e_{13 g} d_{22 g}=0, \\
b_{13 g} c_{22 g}-c_{13 g} b_{22 g}=0, \quad b_{13 g} d_{22 g}-d_{13 g} b_{22 g}=0, \quad b_{13 g} e_{22 g}-e_{13 g} b_{22 g}=0, \\
c_{13 g} d_{22 g}-d_{13 g} c_{22 g}=0, \quad c_{13 g} e_{22 g}-e_{13 g} c_{22 g}=0, \quad d_{13 g} e_{22 g}-e_{13 g} d_{22 g}=0, \\
b_{22 g} c_{23 g}-c_{22 g} b_{23 g}=0, \quad b_{22 g} d_{23 g}-d_{22} b_{23 g}=0, \quad b_{22 g} e_{23 g}-e_{22 g} b_{23 g}=0, \\
c_{22 g} d_{23 g}-d_{22 g} c_{23 g}=0, \quad c_{22 g} e_{23 g}-e_{22 g} c_{23 g}=0, \quad d_{22} e_{23 g}-e_{22 g} d_{23 g}=0, \\
b_{23 g} c_{33 g}-c_{23 g} b_{33 g}=0, \quad b_{23 g} d_{33 g}-d_{23} b_{33 g}=0, \quad b_{23 g} e_{33 g}-e_{23 g} b_{33 g}=0, \\
c_{23 g} d_{33 g}-d_{23 g} c_{33 g}=0, \quad c_{23 g} e_{33 g}-e_{23 g} c_{33 g}=0, \quad d_{23} e_{33 g}-e_{23 g} d_{33 g}=0 .
\end{array}\right.
$$

\section{MiNIMIZATION AND INITIALIZATION OF THE NON-}

\section{LINEAR OBJECTIVE FUNCTION}

To solve the equations (24), in practice there isn't a direct method to solve them. So to solve this problem, we minimized the following non-linear objective function:

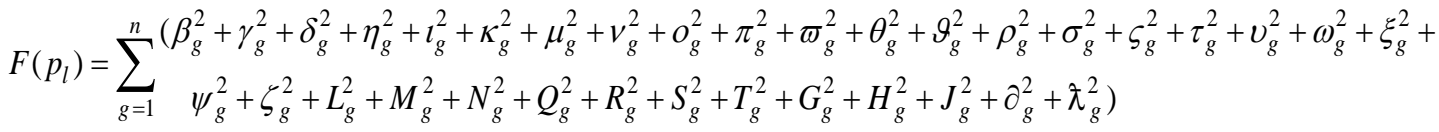

With $\mathrm{n}$ is the number of images, 


$$
\left\{\begin{array}{l}
\beta_{i}=b_{12 g}, \quad \gamma_{i}=c_{12 g}, \quad \delta_{i}=d_{12 g}, \quad \eta_{i}=e_{12 g}, \\
l_{i}=b_{11 g} c_{13 g}-c_{11 g} b_{13 g}, \quad \kappa_{i}=b_{11 g} d_{13 g}-d_{11 g} b_{13 g}, \quad \mu_{i}=b_{11 g} e_{13 g}-e_{11 g} b_{13 g}, \\
v_{i}=c_{11 g} d_{13 g}-d_{11 g} c_{13 g}, \quad o_{i}=c_{11 g} e_{13 g}-e_{11 g} c_{13 g}, \quad \pi_{i}=d_{11 g} e_{13 g}-e_{11 g} d_{13 g}, \\
\varpi_{i}=b_{13 g} c_{22 g}-c_{13 g} b_{22 g}, \quad \theta_{i}=b_{13 g} d_{22 g}-d_{13 g} b_{22 g}, \quad \vartheta_{i}=b_{13 g} e_{22 g}-e_{13 g} b_{22 g}, \\
\rho_{i}=c_{13 g} d_{22 g}-d_{13 g} c_{22 g}, \quad \sigma_{i}=c_{13 g} e_{22 g}-e_{13 g} c_{22 g}, \quad \varsigma_{i}=d_{13 g} e_{22 g}-e_{13 g} d_{22 g}, \\
\tau_{i}=b_{13 g} c_{22 g}-c_{13 g} b_{22 g}, \quad v_{i}=b_{13 g} d_{22 g}-d_{13 g} b_{22 g}, \quad \omega_{i}=b_{13 g} e_{22 g}-e_{13 g} b_{22 g}, \\
\xi_{i}=c_{13 g} d_{22 g}-d_{13 g} c_{22 g}, \quad \psi_{i}=c_{13 g} e_{22 g}-e_{13 g} c_{22 g}, \quad \zeta_{i}=d_{13 g} e_{22 g}-e_{13 g} d_{22 g}, \\
L_{i}=b_{22 g} c_{23 g}-c_{22 g} b_{23 g}, \quad M_{i}=b_{22 g} d_{23 g}-d_{22} b_{23 g}, \quad N_{i}=b_{22 g} e_{23 g}-e_{22 g} b_{23 g}, \\
Q_{i}=c_{22 g} d_{23 g}-d_{22 g} c_{23 g}, \quad R_{i}=c_{22 g} e_{23 g}-e_{22 g} c_{23 g}, \quad S_{i}=d_{22} e_{23 g}-e_{22 g} d_{23 g}, \\
T_{i}=b_{23 g} c_{33 g}-c_{23 g} b_{33 g}, \quad G_{i}=b_{23 g} d_{33 g}-d_{23} b_{33 g}, \quad H_{i}=b_{23 g} e_{33 g}-e_{23 g} b_{33 g}, \\
J_{i}=c_{23 g} d_{33 g}-d_{23 g} c_{33 g}, \quad \partial_{i}=c_{23 g} e_{33 g}-e_{23 g} c_{33 g}, \quad \lambda_{i}=d_{23} e_{33 g}-e_{23 g} d_{33 g} .
\end{array}\right.
$$

To solve the non-linear objective function (25), we used genetic algorithms [29] which require an important initialization step which is to calculate the unknowns assuming certain conditions were verified. Replacing at the end these parameters in the system of equations (24) allows the estimation of the intrinsic camera parameters.

The initialization values are selected such that each parameter of the camera belongs to a specific interval:

TABLE I. VARIATION INTERVAL OF THE CAMERA SETTING

\begin{tabular}{|l|l|}
\cline { 2 - 2 } \multicolumn{1}{c|}{} & $\begin{array}{l}\text { Variation } \\
\text { interval }\end{array}$ \\
\hline $\mathrm{f}_{\mathrm{g}}$ & {$\left[\begin{array}{ll}800 & 2000\end{array}\right]$} \\
\hline$\varepsilon_{g}$ & {$\left[\begin{array}{ll}0 & 1\end{array}\right]$} \\
\hline$\tau$ & {$\left[\begin{array}{ll}0 & 1\end{array}\right]$} \\
\hline$u_{0 g}$ & {$\left[\begin{array}{ll}200 & 300\end{array}\right]$} \\
\hline$v_{0 g}$ & {$\left[\begin{array}{ll}200 & 300\end{array}\right]$} \\
\hline
\end{tabular}

VII. EXPERIMENTATION

\section{1) Real Data}

In this section, a sequence of two images of a checkerboard pattern is simulated to test the performance and robustness of the present approach. After the detection of interests points by the Harris algorithm [26], the matches between each pair of images are determined by the correlation function ZNCC [27]. The pattern is projected in images taken from different views with Gaussian noise of standard deviation $\sigma$, which is added to all image pixels. The projection of the four points in the image planes allows formulating the linear equations, and the solution of these equations gives the projection matrices. The determination of a relationship between the four points and their projections in the image $g$ and the relationships between images of the absolute conic can define a non linear cost function. The minimization of this function by the genetic algorithms [29] allows estimating the intrinsic parameters of the cameras used.

To achieve our theoretical idea and have a practical result, we based on open source tools to offer an application that implements the algorithms used in our article.

The tools used are:

- A robust programming language in the field, objectoriented open source such as Java.

- Sophisticated and open source APIs to solve mathematical complexities such as JAMA, Jscientific and JAI.

- The Swing API for loading images and the realization of different graphical interfaces.

A pair of images is loaded into our application (Figure 3), after the interests points are computed in each image, using the implementation of Harris algorithm (Figure 4), and then the matching of interest points is calculated in the phase matching by implementing the correlation algorithm ZNCC (Figure 5). 


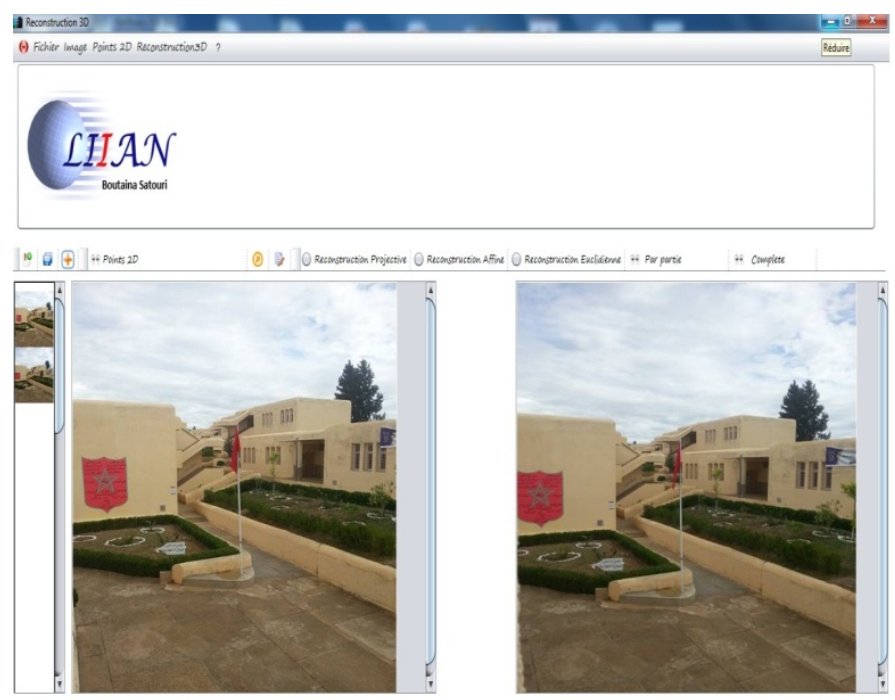

Fig. 3. loading apair of images

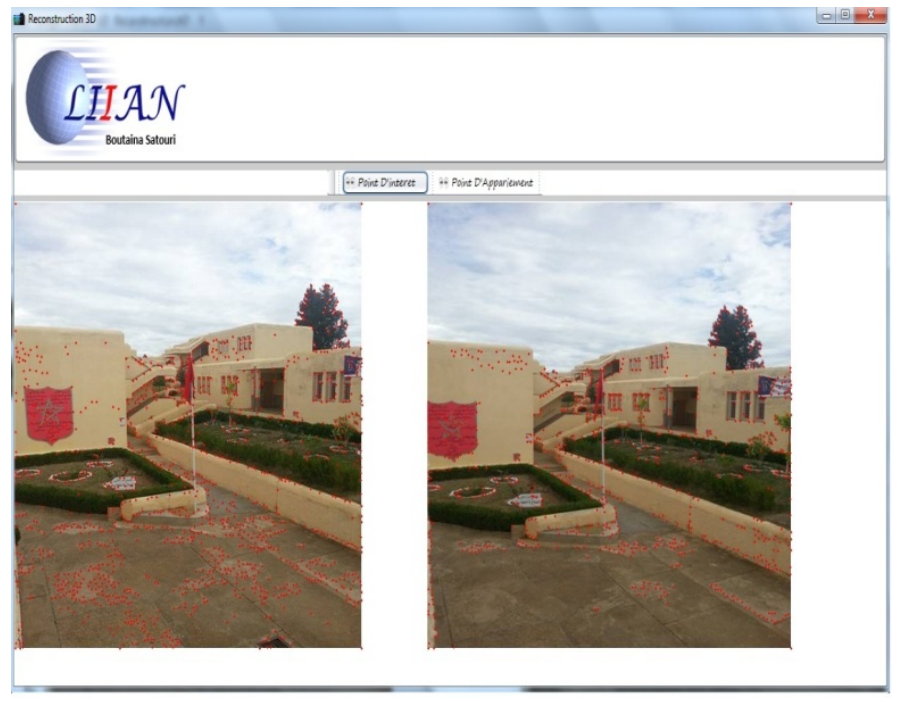

Fig. 4. detection ofInterest pointsfor each image

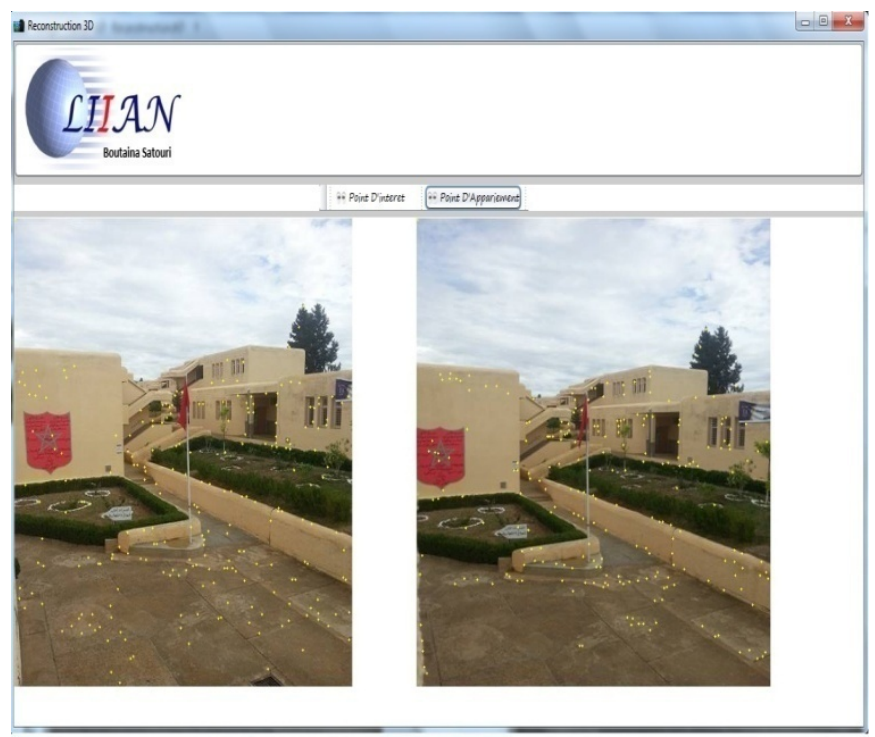

Fig. 5. Matching Interest points by the Correlation algorithm ZNCC 
To estimate the intrinsic parameters of each camera by our approach two phases must be implemented initialization to provide a initial solution and the minimization of cost function (24) to find an optimal solution (Table 2).

\section{RESUlTS AND COMPARAISON OF OUR METHOD WITH OTHER}

TABLE II. THE RESULTS OF THE INTRINSIC CAMERA PARAMETERS ESTIMATED BY THE THREE METHODS

\begin{tabular}{|c|c|c|c|c|c|c|}
\hline & & \multicolumn{5}{|c|}{ Optimal solution } \\
\hline & cameras & $f$ & $\varepsilon$ & $u_{0}$ & $v_{0}$ & $\tau$ \\
\hline \multirow{4}{*}{ 壳 } & Camera1 & 1487 & 0,98 & 257 & 263 & 0,03 \\
\hline & Camera2 & 1480 & 0,97 & 261 & 258 & 0,02 \\
\hline & Camera3 & 1483 & 0,93 & 258 & 261 & 0,05 \\
\hline & Camera4 & 1484 & 0,95 & 260 & 255 & 0,07 \\
\hline \multirow{4}{*}{ 音 } & Camera1 & 1477 & 1 & 263 & 258 & 0 \\
\hline & Camera2 & 1472 & 0,98 & 260 & 263 & 0,12 \\
\hline & Camera3 & 1474 & 0,92 & 254 & 261 & 0,15 \\
\hline & Camera4 & 1471 & 0,94 & 257 & 255 & 0,13 \\
\hline \multirow{4}{*}{ 四 } & Camera1 & 1492 & 0,95 & 240 & 260 & 0,05 \\
\hline & Camera2 & 1490 & 0,92 & 248 & 255 & 0,06 \\
\hline & Camera3 & 1491 & 0,97 & 253 & 258 & 0,04 \\
\hline & Camera4 & 1489 & 0,93 & 252 & 262 & 0,03 \\
\hline
\end{tabular}

In order to show the performance and robustness of our method presented in this paper, the simulation results are compared to those obtained by several efficient methods of Jiang [14], and El akkad [50].

The loading of images is shown in Figure 3, the corner points and the matches between these two images are shown in Figure 4, 5 and the intrinsic parameters estimated by three methods(the present method, El akkad [50], and Jiang's [14]) are shown in Table 2below.

After comparing the results on the synthetic data, the results of the present approach on real data are compared to those obtained by El akkad [50] and Jiang [14] on the same data. The reading and the analysis of the intrinsic camera parameters presented in Table 2show that the results of the present approach are a little different from those obtained by Jiang and El akkad. Therefore, this method provides a robust performance, and it is very close to the other well-established methods. In addition, this method has several advantages: it is based only on the data of the first image without the use the data of the second image for the estimation of the intrinsic camera parameters, the use of any camera and the use of an unknown3D scene.

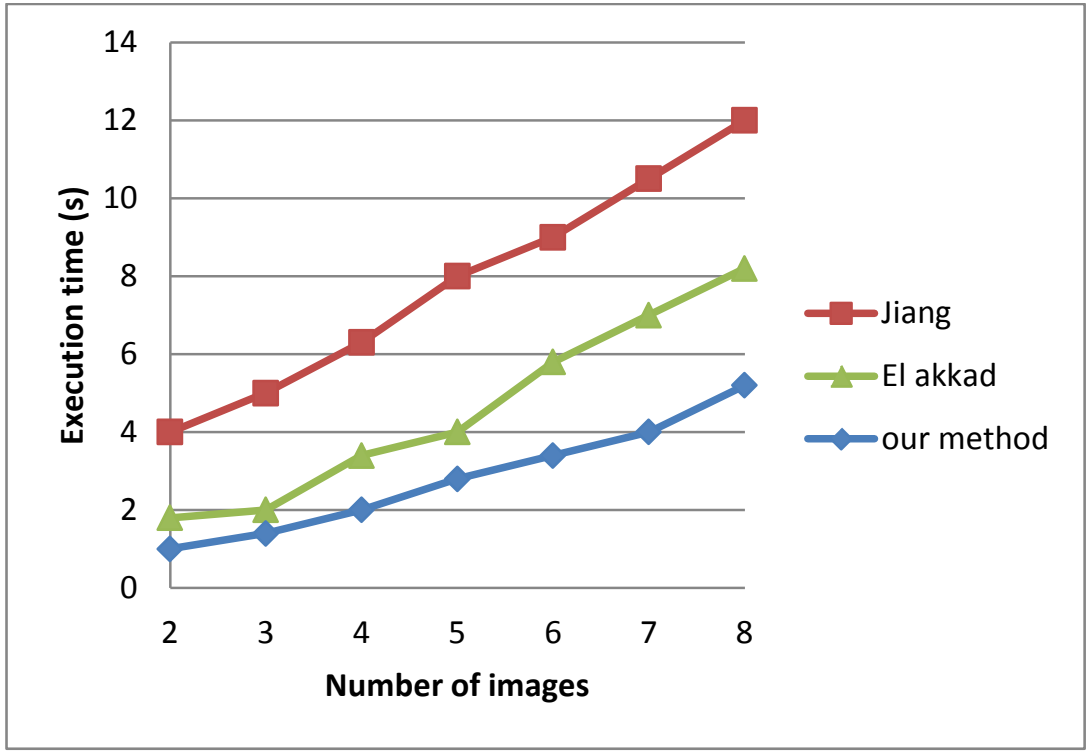

Fig. 6. The execution time according to the number of images

Figure 6 shows that the execution time of the different methods increases, and finally it shows the effect of the use of a large image number.

\section{CONCLUSION}

In this paper, a robust method of camera self-calibration by an unknown three-dimensional scene is presented. The lack of information about the scene requires to be based on mathematical complexities to find the camera settings. This new method is based on the determination of a relationship between two points and oppositely relative to the $\mathrm{X}$ axis in the $3 \mathrm{D}$ scene and their projections in the planes of the images $\mathrm{g}$ and $d$ and between the relationships between the images of the absolute conic. These relationships give a nonlinear cost function, and the minimization of this function provides the intrinsic parameters of the cameras used. Our technology is used to provide more information about the scene which makes the calculation of the parameters in images very simple.

Our method allows easily and without the use or of the data of the second image or the geometrical entities defined between the pair of images estimating the intrinsic parameters of each camera independently from each other. The robustness, the power and the rapidity ofthis method is shown by the results of the experiments andthe simulations conducted.

\section{REFERENCES}

[1] Xiaoqiao Meng, Hua Li and Zhanyi Hu. A New Easy Camera Calibration Technique Based on Circular Points, BMVC2000.

[2] Liangfu Li, Zuren Feng, Yuanjing Feng. Accurate Calibration of Stereo Cameras for Machine Vision. JCS\&T Vol. 4 No. 3, October 2004. 
[3] Jin Sun, Hongbin Gu. Research of Linear Camera Calibration Based on Planar Pattern. World Academy of Science, Engineering and Technology 60, 2009.

[4] Tarek A. Al-Saeed1, Nahed H. Solouma2, and Said M. El-Sherbiny3. Retinal Motion Detection and 3D Structure Recovery From Two Perspective Views. GVIP Special Issue on Medical Image Processing, March, 2006.

[5] A.Elabderrahmani, A.Saaidi and K.Satori. Planar Self-Calibration with Less Constraint. IJCST Vol. 2, Issue 2, June 2011.

[6] A.El abderrahmani, A.Saaidi and K. Satori. Robust Technique for SelfCalibration of Cameras based on a Circle. ICGST-GVIP, Volume 10, Issue 5, December 2010

[7] Triggs, B.: Autocalibration from planar scenes. In: Proceedings of the 5th European Conference on Computer Vision, pp. 89-105 (1998)

[8] Sturm, P.: A case against Kruppa's equations for camera selfcalibration. IEEE Trans. Pattern Anal. Mach. Intell. 22, 1199-1204 (2000)

[9] Saaidi, A., Halli, A., Tairi, H., Satori, K.: Self-calibration using a particular motion of camera. WSEAS Tran. Comput. Res. 3(5), 295-299 (2008)

[10] Zhao, Y., Lv, X.D.: An approach for camera self-calibration usingvanishing-line. Inf. Technol. J. 112, 276-282 (2012)

[11] Zhang, W.: A simple method for 3D reconstruction from twoviews. In: GVIP 05 Conference (2005)

[12] Saaidi, A., Halli, A., Tairi, H., Satori, K.: Self-calibration usinga planar scene and parallelogram. In: ICGST-GVIP, pp. 41-47(2009)

[13] Liu, P., Shi, J., Zhou, J., Jiang, L.: Camera self-calibration usingthe geometric structure in real scenes. In: Proceedings of the ComputerGraphics International, pp. 262-265 (2003)

[14] Baataoui, A., El batteoui, I., Saaidi, A., Satori, K.: Camera selfcalibrationby an equilateral triangle. Int. J. Comput. Appl., 2934(2012)

[15] P.Gurdjos and P.Sturm. Methods and Geometry for Plane-Based SelfCalibration. CVPR, pp. 491-496, 2003.

[16] Manolis I.A. Lourakis and R.Deriche. Camera self-calibration using the kruppa equations and the SVD of the fundamental matrix: the case of varying intrinsic parameters. Technical Report 3911, INRIA, 2000.

[17] Sturm, P.: Critical motion sequences for the self-calibration ofcameras and stereo systems with variable focal length. Image Vis.Comput. 20, 415-426 (2002)

[18] Cao, X., Xiao, J., Foroosh, H., Shah, M.: Self-calibration from turntable sequences in presence of zoom and focus. Comput. Vis.Image Underst. 103(2), 227-237 (2006)

[19] Jiang, Z., Liu, S.: The self-calibration of varying internal camera parameters based on image of dual absolute quadric transformation. In: Information and Automation, Communications in Computer and Information Science, vol. 86, pp. 452-461. Springer, Berlin (2011)

[20] Shang, Y., Yue, Z., Chen, M., Song, Q.: A new method of camera self-calibration based on relative lengths. Inf. Technol. J. 11(3), 376-379 (2012)

[21] Jiang, Z., Liu, S.: Self-calibration of varying internal camera parameters algorithm based on quasi-affine reconstructio J. Comput. 7(3), 774-778 (2012)

[22] Zhao, Y., Hu, X., Lv, X., Wang, H.: Solving the camera intrinsic parameters with the positive tri-prism based on the circular points. Inf. Technol. J. 11(7), 926-930 (2012)

[23] Gao, Y., Radha, H.: A multistage camera self-calibration algorithm. In: Proceedings of IEEE International Conference on Acoustics, Speech, and Signal Processing (ICASSP), pp. 537-540 (2004)

[24] El akkad, N., Saaidi, A., Satori, K.: Self-calibration based on a circle of the cameras having the varying intrinsic parameters. In: Proceedings of IEEE International Conference on Multimedia Computing and Systems, pp. 161-166 (2012)

[25] Hemayed, E.E.: A survey of camera self-calibration. In: Proceedings of the IEEE Conference on Advanced Video and Signal BasedSurveillance, pp. 351-357 (2003)

[26] C. Harris and M. Stephens, “ A combined corner and edge detector,” In Alvey Vision Conference, 1988.
[27] Chambon, S., Crouzil, A.: Similarity measures for image matching despite occlusions in stereo vision. Pattern Recognit. 44(9), 20632075 (2011)

[28] Torr, P.H.S., Murray, D.W.: The development and comparison of robust methods for estimating the fundamental matrix. Int. J.Comput. Vis. 24, 271-300 (1997)

[29] J. H., Holland. Adaptation in Natural and Artificial Systems. University Bibliographieof Michigan Press: Ann Arbor, 1975.

[30] M.J. Brooks, W. Chojnacki, and L. Baumela. Determining the egomotion of an uncalibrated camera from instantaneous optical flow. Journal of the Optical Society of America, 14(10), October 1997.

[31] M.J. Brooks, L. de Agapito, D.Q. Huynh, and L. Baumela. Direct methods for self-calibration of a moving stereo head. In B. Buxton and R. Cipolla, editors, Proceedings of the 4th European Conference on Computer Vision, Cambridge, England, volume 1065 of Lecture Notes in Computer Science, pages 415.426. Springer-Verlag, April 1996.

[32] M. Armstrong, A. Zisserman, and R. Hartley. Self-calibration from image triplets. In B. Buxton and R. Cipolla, editors, Proceedings of the 4th European Conference on Computer Vision, Cambridge, England, volume 1064 of Lecture Notes in Computer Science, pages 3.16. Springer- Verlag, April 1996.

[33] C. Wiles and M. Brady. Ground plane motion camera models. In B. Buxton and R. Cipolla, editors, Proceedings of the 4th European Conference on Computer Vision, Cambridge, England, volume 1065 of Lecture Notes in Computer Science, pages 238.247. Springer-Verlag, April 1996.

[34] P.Sturm, A case against Kruppa's equations for camera self-calibration, IEEE Transactions on Pattern Analysis and Machine Intelligence, Vol. 22, Issue 10, pp. 1199-1204, October 2000.

[35] R. Hartley, A. Zisserman, Multiple View Geometry in Computer Vision, seconde édition, Cambridge University Press, ISBN 0-521-54051-8, 2004.

[36] Houman Rastgar, Eric Dubois, Liang Zhang. Random Sampling Nonlinear Optimization for Camera Self-calibration with Modeling of Intrinsic Parameter Space. Advances in Visual Computing, 6th International Symposium, ISVC 2010, Las Vegas, NV, USA, November 29 - December 1, 2010, Proceedings, Part III, pp 189-198.

[37] B. Boudine, A. El abderrahmani, A. Saaidi et K. Satori. Détection des points d'intérêt entre les détecteurs Harris, Sift et Surf. Communication au Workshop en Imagerie, Systèmes et Applications. Mai 23-24, 2013, FP-Taza Maroc.

[38] Adlane Habed, Kassem Al Ismaeil, David Fofi. A New Set of Quartic Trivariate Polynomial Equations for Stratified Camera Self-calibration under Zero-Skew and Constant Parameters Assumptions. Computer Vision- ECCV 2012 Lecture Notes in Computer Science Volume 7577, 2012, pp 710-723.

[39] Adlane Habed, Tarik Elamsy, Boubakeur Boufama. Combining Mendonça-Cipolla Self-calibration and Scene Constraints. Advances in Image and Video Technology Lecture Notes in Computer Science Volume 7088, 2012, pp 168-179.

[40] B. Triggs, Autocalibration and the Absolute Quadric, Proceedings of the 1997 Conference on Computer Vision and Pattern Recognition, Porto Rico, USA, p. 609-614, 17-19 juin 1997.

[41] A. Vladés, J. Ronda and G. Gallego, The Absolute Line Quadric And Camera Autocalibration, International Journal of Computer Vision, vol. 66, n 3, p. 283-303, mars 2006.

[42] R. Hartley, A. Zisserman, Multiple View Geometry in Computer Vision, seconde édition, Cambridge University Press, ISBN 0-521-54051-8, 2004.

[43] P. Sturm, On Focal Length Calibration from Two Views, Proceedings of the 2001 IEEE Computer Society Conference on Computer Vision and Pattern Recognition, Kauai, Hawaï, vol. 2, p. 145-150, 8-14 décembre 2001.

[44] Y. Li, W. K. Tang, Y. S. Hung Stratified Self Calibration and Metric Reconstruction for Zooming/Refocusing Circular Motion Sequences. Journal of Mathematical Imaging and Vision. May 2008, Volume 31, Issue 1 , pp 105-118.

[45] Ze-tao Jiang, Shan-chao Liu. The Self-calibration of Varying Internal Camera Parameters Based on Image of Dual Absolute Quadric 
Transformation. Information and Automation Communications in Computer and Information Science Volume 86, 2011, pp 452-461.

[46] Zetao Jiang, Shutao Guo, Lianggang Jia. Sequences Images Based Camera Self-calibration Method. Intelligent Science and Intelligent Data Engineering Lecture Notes in Computer Science Volume 7202, 2012, pp 538-545.

[47] Jong-Eun Ha, Dong-Joong Kang. Initialization Method for the SelfCalibration Using Minimal Two Images. Computational Science and Its Applications - ICCSA 2004 Lecture Notes in Computer Science Volume 3046, 2004, pp 915-923.
[48] Javier Civera, Andrew J. Davison, José María Martínez Montiel. Selfcalibration. Structure from Motion using the Extended Kalman Filter Springer Tracts in Advanced Robotics Volume 75, 2012, pp 111-122.

[49] Thorsten Thormählen, Hellward Broszio, Patrick Mikulastik. Robust Linear Auto-calibration of a Moving Camera from Image Sequences. Computer Vision - ACCV 2006. Lecture Notes in Computer Science Volume 3852, 2006, pp 71-80.

[50] N. El akkad, M. Merras, A. Saaidi and K. Satori. Camera SelfCalibration with Varying Intrinsic Parameters by an Unknown Three-Dimensional Scene. The Visual Computer (Springer). Vol. 30, No. 5,pp. 519-530, 2014. 